\title{
Comparison of Colposcopic Biopsy Results of Non-HPV 16/18 Oncogenic Type Positive Patients
}

\author{
(1) Nazı Aylin Vural ${ }^{1}$, (1) Gizem Nur Köyann ${ }^{2}$, (1) Ozan Karadeniz ${ }^{3}$, (1) Şenay Erdoğan Durmuş ${ }^{4}$, \\ (1) Özge Kocaman ${ }^{4}$, @ Hasan Turan ${ }^{1}$ \\ ${ }^{1}$ University of Health Sciences Turkey, Başakşehir Çam and Sakura City Hospital, Clinic of Gynecology and \\ Gynecologic Oncology, İstanbul, Turkey \\ 2University of Health Sciences Turkey, Başakşehir Çam and Sakura City Hospital, Clinic of Obstetrics and \\ Gynecology, İstanbul, Turkey \\ ${ }^{3}$ University of Health Sciences Turkey, Kanuni Sultan Süleyman Training and Research Hospital, Clinic of Obstetrics \\ and Gynecology, İstanbul, Turkey \\ ${ }^{4}$ University of Health Sciences Turkey, Başakşehir Çam and Sakura City Hospital, Clinic of Pathology, İstanbul, \\ Turkey
}

\section{What is known on this subject?}

High-risk human papillomavirus is the most common cause of cervical cancer worldwide.

\section{What this study adds?}

If gynecologists follow the algorithms recommended by guidelines, there is a risk of misdiagnosing cervical intraepithelial neoplasia $2+$ lesions in $4 \%$ of those patients

\section{ABSTRACT}

Objective: This study aimed to evaluate and compare the risk in detecting cervical intraepithelial neoplasia (CIN) $2+$ or higher lesions by performing immediate colposcopy in patients with positive high-risk non-human papillomavirus (HPV) 16/18 subtypes, regardless of their cytology results.

Material and Methods: A total of 264 patients with HPV-positive subtypes, aged 20-65 years, with any type of cervical cytology results were included in the study. A liquid-based cytologic cervical cancer screening with HPV testing was carried out between November 2020 and May 2021. Cytological specimens were classified according to the Bethesda system (2014), and HPV identification was analyzed with Cobas 4800 system. Colposcopy-guided endocervical curettage and endometrial biopsy were performed.

Results: A total of 123 patients had HPV non-16/18 oncogenic types, wherein 34 (69.3\%) had no dysplasia, 9 (18.3\%) had CIN 1, and 2 (4.08\%) had CIN 2-3.

Conclusion: Colposcopic evaluation may be considered in cases of non-16/18 high-risk HPV subtypes with abnormal cytologic results. Among the patients who had negative cervical cytology and positive non-HPV-16/18, 4.08\% were women with CIN 2-3. Following the algorithm according to the guidelines, there will be a risk of $4.08 \%$ of misdiagnosing CIN $2+$ lesions by gynecologists. Organizing large-scale randomized controlled studies will help in understanding the meaning or importance of this topic.

Keywords: Colposcopy, cytology, genotyping, human papillomavirus subtypes

Address for Correspondence: Nazlı Aylin Vural MD, University of Health Sciences Turkey, Başakşehir Çam and Sakura City Hospital, Clinic of Gynecology and Gynecologic Oncology, İstanbul, Turkey

Phone: +90 5334877893 E-mail: draylinvural@gmail.com ORCID ID: orcid.org/0000-0003-0493-5439 Received: 15.04.2021 Accepted: 17.06.2021 


\section{Introduction}

High-risk human papillomavirus (HR-HPV) is the most common cause of cervical cancer worldwide (1). According to the National Cancer Institute Centers for Disease Control and Prevention, HPV infection is responsible for $>90 \%$ of cervical cancer cases worldwide. Almost 200,000 women are estimated to be diagnosed with precancerous cervical lesions or abnormal cells, which may lead to cancer. Of those, 11,000 women have lesions that progressed into cervical cancer as a result of chronic HPV infection. Unfortunately, over 4,000 women died because of this disease (2).

Randomized controlled trials have highlighted that HPV screening tests alone compared with cytologic interpretation were thought to facilitate an objective way of providing better protection against high-grade cancer precursors, such as cervical intraepithelial neoplasia (CIN $2+$ and $\mathrm{CIN} \mathrm{3+)}$ ) and cervical cancer, than cytology tests, respectively $(1,2)$.

According to the American Society for Colposcopy and Cervical Pathology (ASCCP) 2019 guidelines, co-testing after 1 year has been recommended in the presence of non-HPV $16 / 18$ subtypes with normal cytology for women who are 3065 years old (3). The difference between the 2012 and 2019 ASCCP guidelines is that in 2019, all positive primary HPV screening tests were recommended, regardless of genotype, to have additional reflex triage testing from the same laboratory specimen. The specificity of HPV testing in CIN $2+$ and higher lesions decreased by $2-4 \%$ compared with cytology testing, and HPV testing alone would direct patients to overtreatment and over referral (4). Therefore, reflex triage testing is needed (5).

Risk-based management recommendations are the main difference that comes out at the 2019 ASCCP guidelines. The combination of present HPV results in history (including unknown history) for the surveillance, patient treatments, and colposcopy referrals immediately determined the risk for CIN 3+.

A Turkish nationwide study (6) stated that the positive predictive value for the risk of $\geq$ CIN 2 lesions of some other types of HPV, such as $33,31,35$, and 45 , in addition to HPV $16 / 18$ is approximately $10 \%$. Nevertheless, the current literature contains limited data on the risk of $\geq$ CIN 2 lesions in cases with non-HPV 16/18 high-risk types regardless of the cytology results.

The recent cervical cancer screening program is mainly based on searching or detecting the chronic HR-HPV subtypes. Direct colposcopic evaluation is recommended when the HPV 16/18 subtypes are detected (7). The prevalence of these HPV
16/18 subtypes may decrease due to HPV vaccination. In addition, the ATHENA study is similar to the study of Keiser and NHANES, without a sharp increase or decrease in the prevalence of HR-HPV (8) because of the underestimation in managing other high-risk non-16/18 HPV subtypes. The contribution of colposcopic evaluation toward the management of non-16/18 HPV high-risk cases is unclear.

This study aimed to evaluate and compare the risk of detecting CIN $2+$ or higher lesions by performing colposcopy in patients with a positive high risk of non-16/18 HPV subtypes, regardless of their cytology results.

\section{Material and Methods}

This retrospective study was conducted between November 2020 and May 2021 in a tertiary center located in İstanbul at the Obstetrics and Gynecology Department, Oncology Division, after obtaining approval from Çam and Sakura City Hospital Human Research Ethics Committee (approval no: 2021.07.156). A total of 264 patients, aged 2065 years, who were screened for cervical cancer with liquidbased cytology and HPV testing were included in this study. The study population was grouped according to their HPV 16/18 test results and previous history of abnormal cervical cytology or cancer. After obtaining informed consent from all participants, the colposcopy and biopsy procedures were performed. Patients who rejected the biopsy procedure and inadequate biopsy samples were excluded from the study. A total of 123 patients were retrospectively evaluated (Figure 1).

All cervical cytology samples were implemented using the Bethesda 2014 system (Surepath). HPV type identification was analyzed using Cobas 4800 for 14 types of HR-HPV DNA $(16,18$, $31,33,35,39,45,51,52,56,58,59,66$, and 68). Colposcopy was performed on patients who had HR-HPV-DNA types regardless of cytology results. Patients were divided into two categories based on their cytology results as "negative cytology/non-HPV 16/18 positive types" and "positive cytology/non-HPV 16/18 positive types."

Histological examination with colposcopy was performed in all patients following the procedure of 3-5\% acetic acid solution on the cervix and upper vagina. The evaluation of the colposcopy findings is based on the solemnity of acetowhite lesions, an extension of lesion margins, and vascularity within the acetowhite lesion. Subsequently, Lugol's solution was applied using an injector by direct installation in the same way with acetic acid. A biopsy of at least four parts from the Lugol negative and acetowhite positive areas was conducted. In the absence of abnormal lesions, a random biopsy was performed. All colposcopy-guided biopsies and 
large loop electrosurgical excision (LEEP) procedures were performed by specialists in the gynecological oncology division. Endocervical curettage (ECC) was performed during the colposcopy-guided biopsy procedure. LEEP or conization procedures were executed in case of initial biopsy results with substantiated high-grade cervical lesions (CIN 2-3) or carcinoma in situ. The International Federation for Cervical Pathology and Colposcopy 2011 nomenclature was used for the transformation zone classification.

\section{Statistical Analysis}

All data were statistically analyzed using the SPSS software version 21. Descriptive statistics were used for demographic data. Fisher's Exact test and chi-squared association test were used for categorical data. The Student's t-test was performed for continuous data. A $p$ value of $<0.05$ was considered statistically significant. Age, obstetric history, contraceptive method, smoking, cytology results, ECC, LEEP/conization, and cervical pathology results were all recorded.
According to the cervical biopsy results, those with lowgrade dysplasia were considered CIN 1, and those with highgrade dysplasia were considered CIN 2-3.

\section{Results}

A total of 123 patients with non-16/18 HR-HPV subtypes were included and divided into two groups: Negative cytology group and positive cytology group. The mean ages of patients in the negative cytology and positive cytology groups were 40.8 (24-60) years and 40.4 (20-65) years, respectively $(p=0.40)$. No significant difference was found in the demographics (Table 1).

All patients underwent colposcopy, and a colposcopyguided cervical biopsy was performed in 45 (91.8\%) patients (Table 2).

Endocervical canal curettage biopsy was performed in 36 (73.4\%) patients of the negative cytology group, wherein all resulted in chronic cervicitis/inflammation (Table 3).

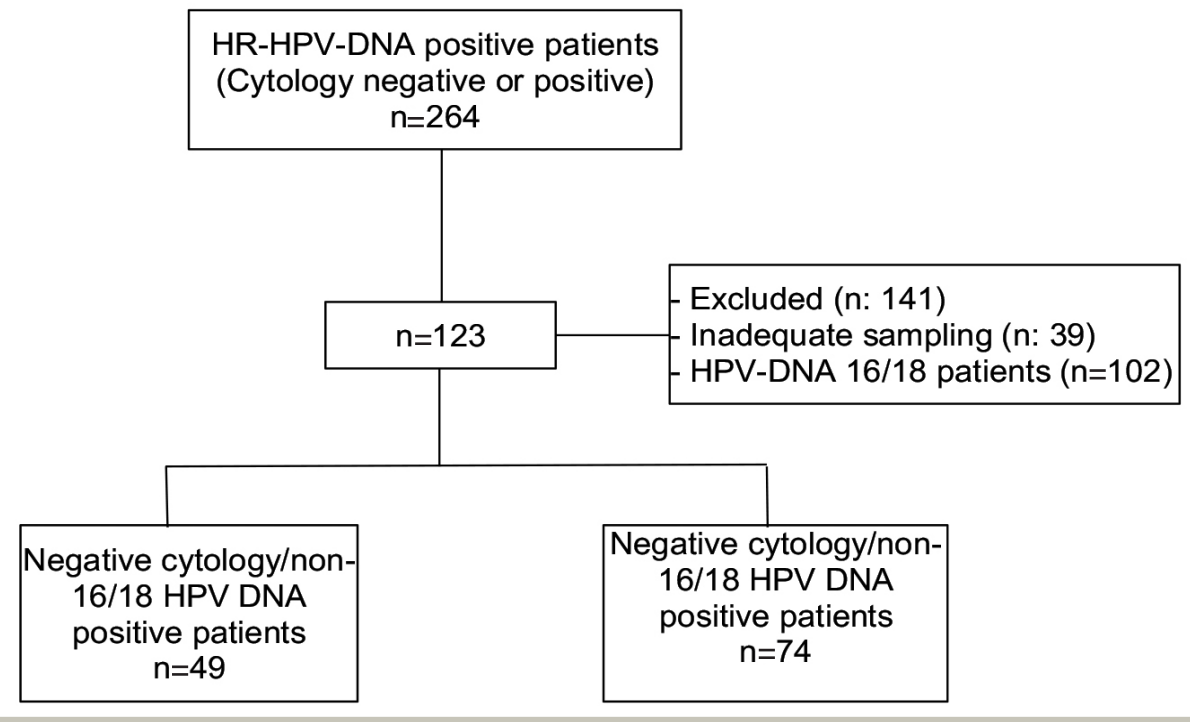

Figure 1. Flow chart of non-HPV-DNA 16/18 positive patient with negative or positive cytology HR-HPV: High-risk human papillomavirus

Table 1. Comparison of demographics of patients with positive non-16/18 HPV DNA with negative or positive cytology

\begin{tabular}{llll|} 
& Negative cytology $(\mathrm{n}=49)$ & Abnormal cytology $(\mathbf{n}=74)$ & $\mathbf{p}$ value* \\
\hline Age (mean) & $40.8(24-60)$ & $40.4(20-65)$ & 0.40 \\
\hline Gravida (mean) & 2.38 & 2.43 & 0.45 \\
\hline Parite (mean) & 1.93 & 1.9 & 0.45 \\
\hline Smoker & $11(22.4 \%)$ & $16(21.6 \%)$ & 0.82 \\
Contraceptive pill usage & $1(2.05 \%)$ & $2(2.70 \%)$ & 0.06 \\
HPV: Human papillomavirus & & & \\
\hline
\end{tabular}




\begin{tabular}{|c|c|c|c|}
\hline & Negative cytology $(n=45)$ & Abnormal cytology $(n=73)$ & p value \\
\hline Chronic cervicitis, inflammation & $34(69.3 \%)$ & $40(54.05 \%)$ & \multirow{3}{*}{0.02} \\
\hline Low-grade dysplasia, CIN 1 & $9(18.3 \%)$ & $17(22.97 \%)$ & \\
\hline High-grade dysplasia (CIN 2-3) & $2(4.08 \%)$ & $16(21.6 \%)$ & \\
\hline CIN: Cervical intraepithelial neoplasia & & & \\
\hline
\end{tabular}

\begin{tabular}{|c|c|c|c|}
\hline & Negative cytology $(n=36)$ & Abnormal cytology $(n=63)$ & p value \\
\hline Chronic cervicitis, inflammation & $36(100 \%)$ & 60 & \multirow{3}{*}{0.94} \\
\hline Low-grade dysplasia, (CIN 1) & - & 2 & \\
\hline High-grade dysplasia (CIN 2-3) & - & 1 & \\
\hline CIN: Cervical intraepithelial neoplasia & & & \\
\hline
\end{tabular}

\section{Discussion}

This retrospective cohort study demonstrated that non16/18 HR-HPV subtypes cause nearly $15 \%$ of CIN 2+ lesions. This probability increased to $21.6 \%$ with abnormal cytologic results. The subtype analysis of the non-16/18 HR-HPV group in cases, which resulted in CIN 2+ lesions, was not defined.

Gultekin et al. (6) stated that the ratio of CIN 2+ lesions for the non-16/18 HPV subtypes was reported to be nearly $17 \%$, which were nearly $24 \%$ of the HPV $16 / 18$ subtypes. They put forward the necessity of reflex cytologic tests for the non-16/18 HPV subtypes to prevent unnecessary colposcopic evaluations. Our results correspond with this research. Our study found that the detection rate of CIN 2+ lesions statistically increased with abnormal cytology.

Aydoğmuş and Aydoğmuş (9) reported that the ratio of CIN $2+$ lesions in cases of normal cytology results with non-16/18 HPV subtypes was $15.6 \%$. Conversely, in another research, this ratio was reported as $0.01 \%$ (10). Our research detected CIN $2+$ lesions $4 \%$ of patients with negative cytology. Interestingly, Çöl Madendağ et al. (11) reported that the detected CIN 2+ lesions with normal cytology were higher in cases of non-16/18 HPV subtypes, contrary to 16/18 HPV subtypes. Yalcin et al. (12) stated that the colposcopic evaluation of the normal cytologic results of the HPV 16 cases did not increase the detection rate of cervical cancer. Conflicting results are reported in the literature in cases of negative cytology results with positive non-16/18 HPV subtypes. The regional differences and the possible effect on the virulence or the behavior of the non16/18 HPV subtypes may cause these conflicting results.
The reported cases demonstrated that even with the normal cytology results, non-16/18 HPV subtypes may cause higher dysplastic lesions at the uterine cervix $(6,9,10)$. Colposcopic evaluation seems logical in cases with abnormal cytology results with non-16/18 HR-HPV subtypes. Colposcopic evaluation is considered to be a burden in the healthcare system; however, as a new and modern gynecologic oncology department, our oncology experts can carry out colposcopic examinations. Colposcopic evaluations are easily accessible and applicable. This is the major strength of this research. This research provides information about the management of the non-16/18 HR-HPV-positive cases.

\section{Study Limitations}

One of the limitations of this study is the colposcopic evaluation. Reid's colposcopic index was not found in the patients' colposcopy reports. Retrospective design and limited data is also a limitation of this study.

\section{Conclusion}

Organizing large-scale randomized controlled studies would be beneficial in understanding the importance of this topic.

\section{Ethics}

Ethics Committee Approval: Çam and Sakura City Hospital Human Research Ethics Committee approved (approval no: 2021.07.156).

Informed Consent: Approval received.

Peer-review: Externally peer-reviewed. 


\section{Authorship Contributions}

Surgical and Medical Practices: Ș.E.D., Ö.K., H.T., Concept: H.T., Design: N.A.V., H.T., Data Collection or Processing: N.A.V., G.N.K., H.T., Analysis or Interpretation: N.A.V., G.N.K., O.K., Literature Search: N.A.V., G.N.K., O.K., Writing: N.A.V., G.N.K., O.K., S.E.D.
Conflict of Interest: No conflict of interest was declared by the authors.

Financial Disclosure: The authors declared that this study received no financial support.

\section{REFERENCES}

1. Ronco G, Dillner J, Elfström KM, et al. Efficacy of HPV-based screening for prevention of invasive cervical cancer: follow-up of four European randomised controlled trials. Lancet 2014;383:524532. Erratum in: Lancet 2015;386:1446.

2. Arbyn M, Ronco G, Anttila A, et al. Evidence regarding human papillomavirus testing in secondary prevention of cervical cancer. Vaccine 2012;(30 Suppl 5):F88-99. Erratum in: Vaccine 2013;31:6266.

3. Massad LS, Einstein MH, Huh WK, et al. 2012 updated consensus guidelines for the management of abnormal cervical cancer screening tests and cancer precursors. J Low Genit Tract Dis 2013;17(5 Suppl 1):S1-S27. Erratum in: J Low Genit Tract Dis 2013;17:367.

4. Uijterwaal MH, Polman NJ, Van Kemenade FJ, et al. Five-year cervical (pre)cancer risk of women screened by hpv and cytology testing. Cancer Prev Res (Phila) 2015;8:502-508.

5. Cox JT. Management of atypical squamous cells of undetermined significance and low-grade squamous intra-epithelial lesion by human papillomavirus testing. Best Pract Res Clin Obstet Gynaecol 2001;15:715-741.

6. Gultekin M, Dundar S, Keskinkilic B, et al. How to triage HPV positive cases: results of four million females. Gynecol Oncol 2020;158:105111.

7. US Food and Drug Administration (FDA). FDA approves first hu- man papillomavirus test for primary cervical cancer screening. Silver
Spring, MD: FDA; 2014. Available : www.eve-medical.com/fda-approves-first-human-papillomavirus-test-for-primary-cervical-cancer-screening/ Accessed: January 1, 2018.

8. Wright TC Jr, Stoler MH, Behrens CM, Apple R, Derion T, Wright TL. The ATHENA human papillomavirus study: design, methods, and baseline results. Am J Obstet Gynecol 2012;206:46.e1-46.e11.

9. Aydoğmuş $\mathrm{H}$, Aydoğmuş S. Comparison of Colposcopic biopsy results of patients who have cytomorphological normal but HPV 1618 or other high-risk HPV subtypes positive. Asian Pac J Cancer Prev 2019;20:417-420.

10. Karaca I, Öztürk M, Comba C, et al. Immediate biopsy of cervical cytology-negative and non-HPV-16/18 oncogenic types positive patients. Diagn Cytopathol 2018;46:326-330.

11. Çöl Madendağ I, Madendağ Y, Şahin E, Eraslan Şahin M, Açmaz $\mathrm{G}$. Is colposcopic examination necessary in women with high risk HPV without HPV16/18? The Journal of Gynecology - Obstetrics and Neonatology 2018;15:102-105.

12. Yalcin I, Sari ME, Sahin H, Gultekin M, Gungor T, Meydanli MM. Colposcopic biopsy findings among women with either HPV-16 only or HPV-18 only who have normal cervical cytology. Int J Gynaecol Obstet 2018;143:300-305. 